Vol. 8(4), pp. 36-44, April 2016

DOI: $10.5897 / I J M M S 2015.1171$

Article Number: 2C3210A58484

ISSN 2006-9723

Copyright (C) 2016

Author(s) retain the copyright of this article

http://www.academicjournals.org/IJMMS
International Journal of Medicine and Medical Sciences

\title{
Assessment of the quality of mobile voluntary counseling and testing services in Addis Ababa and Adama organization for social services for AIDS (OSSA) branches, Ethiopia
}

\author{
Tigist Tamirayehu ${ }^{1}$, Tirhas Tadesse ${ }^{2}$ and Zelalem Kebede ${ }^{1 *}$ \\ ${ }^{1}$ Police Medical Professionals Training Institute, Department of Public Health, Ethiopian Police Universit College. \\ ${ }^{2}$ Department of Public Health, Yekatit 12 Hospital Medical College, Ethiopia.
}

Received July, 2015; Accepted 15 September 2015

\begin{abstract}
Mobile or outreach type of voluntary counseling and testing approach is a useful way to reach high risk groups and rural population that are far away from facility based voluntary counseling and testing (VCT) service. Little has been done to assess the quality of mobile VCT services in Ethiopia. This study aims to assess the quality of mobile VCT services and client satisfaction in the cities of Addis Ababa and Adama. This study used both qualitative and quantitative study designs. Structured questionnaire was administered to 279 client respondents. The qualitative data was analyzed by thematic areas, and the quantitative data was analyzed and interpreted by using statistical packages for social sciences (SPSS) package of version 13.0. Overall service satisfaction of the clients was very high in this study. The competence of the counselors was good. Short duration of counseling time, inconvenience of counseling rooms, high number of clients seen by the counselors amongst other factors were findings from the qualitative study that can affect the quality of the mobile VCT services. To improve the promotional activities, the referral linkages and the feedback problem, there should be work integration with government health institutions, non-governmental organization (NGOs) and kebele (the smallest administrative unit) HIV focal persons.
\end{abstract}

Key words: Quality, satisfaction, quality of VCT counselors, providers, client, quality of VCT site,

\section{INTRODUCTION}

Voluntary human immunodeficiency virus (HIV) counseling and testing is one of the most rapidly expanding HIV program services in the world. Propelling the increased demand is the recognition of counseling and testing role in preventing new HIV infections, and increasing access to care and treatment. In order to make voluntary counseling and testing (VCT) services more widely available to groups who do not routinely attend health facilities, mobile VCT clinics have been developed in some settings. Mobile VCT is an effective way to bring

*Corresponding author. E-mail:welzol@yahoo.com.

Author(s) agree that this article remain permanently open access under the terms of the Creative Commons Attribution License 4.0 International License 
services to high-risk groups. A study has revealed that there is a very high demand for VCT services, when the services are provided in the community (WHO 2002; WHO 2009; Joseph et al., 2006).

The use of testing in Africa however is still low. The 2009 estimates of data and statistics in sub-Saharan Africa indicate that a median of $22 \%$ of people age 15 to 49 years old in the general population have been tested for HIV. Even though the data shows that there is a slight improvement in the uptake of VCT service, there are still many people living with HIV testing and counseling only when they already have advanced clinical disease (Joseph et al., 2006; WHO, UNICEF and UNAIDS 2009).

Currently in Ethiopia, most people remain unaware of their HIV status due to various reasons. According to the 2008 data, HIV testing utilization of the country is $12.1 \%$ in the general population. However, with the development of affordable and effective medical care for people living with HIV, demand for testing is increasing rapidly, creating urgent need to increase access. The availability of HIV counselling and testing (HCT) services in Ethiopia has not been expanded equally all over the country, and even when available, uptake has been relatively low (UNICEF and UNAIDS 2009; CDC 2004).

This study has mainly focused on the outreach or mobile VCT services. The mobile VCT service was started for the first time in this country in September, 9, 2005, in Meskel square, by OSSA Ethiopia. The "mobile unit" can be a van/caravan or a truck which offers VCT in situ, and has a schedule of places which it visits at pre-advertised times. This approach has been used to access "hard-toreach" populations who do not visit formal health settings, such as homeless people, sex workers or injecting drug users (IDUs). Mobile VCT service is a very useful approach for increasing the uptake of VCT service. Thus, addressing the factors that can affect the quality of the service is very important (CDC 2004; Asingwire (2004).

Little is known about the quality of mobile VCT service in this country. Hence, the purpose of this study is to assess whether there are problems that can affect the quality of mobile VCT services in the selected sites.

\section{METHODOLOGY}

\section{Study design, period and area}

Both qualitative and quantitative types of study designs were used in this study. By using quantitative study design, client satisfaction was assessed, and as part of the qualitative study design, the operational aspect of VCT and the technical competence of providers were assessed. The study was conducted in mobile VCT centers of OSSA in Addis Abeba, the Capital of Ethiopia and Adama, town found about $100 \mathrm{Km}$ to the east of the capital.

\section{Study population}

The study subjects included in the study were VCT managers, service providers and clients who had come at the time of data collection in the selected mobile sites. The required sample size was determined by using single population proportion formula. A prevalence of $79 \%$ client satisfaction had been taken from a study done in Addis Ababa (Dawit 2006).

\section{Variables}

The dependent variable was client satisfaction and the independent variables were duration of the counseling session, waiting time to see a counselor, waiting time to take test results, convenience of time and place of service delivery, confidentiality, charge-free service and socio demographic variables.

\section{Sampling technique}

The required sample size was determined by using single population proportion formula. A prevalence of $79 \%$ client satisfaction had been taken from a study done in Addis Ababa (Dawit 2006).

Level of significance $=0.05$

Marginal of error $(d)=5 \%$

Non-response rate $=10 \%$

By using the formula for calculating the sample size $(n)$

$\mathrm{N}=\frac{(\mathrm{Za} / 2)^{2} \mathrm{P} \times(1-\mathrm{P})}{\mathrm{d}^{2}}$

$\mathrm{n}=(1.96)^{2} \times .79 \times .21 /(0.05)^{2}$

$\mathrm{n}=254$

With the above assumptions, the overall sample size became $=254+25=279$

According to the data found from both areas, the number of clients seen in one month before the data collection period (January 2010) was found to be 835 and 1032 in Addis Ababa and Adama OSSA branches, respectively. Based on this, the sample size was divided proportionally into the two study areas and became 126 and 153 study subjects to Addis Ababa and Adama, respectively. Since it was very difficult to cover all mobile VCT sites which are found in this country, within limited time and resources available for the study, only mobile VCT sites found in Addis Ababa and Adama city were selected by using convenience method. The study subjects had been selected by using quota sampling method until the required sample size was fulfilled during the study period.

\section{Data collection}

Ethical clearance was obtained from Addis Continental Institute of Public Health (ACIPH) and University of Gondar (UoG), and was communicated with the concerned bodies of the two study areas. During the data collection period in Adama OSSA branch, the service providers were rendering the mobile VCT service $20 \mathrm{Km}$ away from Adama city in a small town called Welenchiti. And in Addis Ababa OSSA branch they were rendering the service at Sidest kilo and Gofa areas. Therefore, the data was collected from these areas.

The data was collected from VCT managers, service providers (counselors and lab technicians) and clients who had come for VCT service at the time of data collection period. There were four data collectors for both study areas. They had the qualification of $10+3$ diploma level and oneday training was given to them by the principal investigator. A structured questionnaire was adopted from 
UNAIDS materials to assess client satisfaction. Before the administration of the structured questionnaire, pre-test had been done on VCT clients to ensure the understanding of the respondents. For clients who couldn't read and write, the questionnaires were filled by the data collectors and the remaining questionnaires were self administered. By using interview guides, the principal investigator conducted in depth interviews with clients, VCT managers and service providers. There were two counseling session observations in each mobile VCT sites which was undertaken by the principal investigator. To ensure quality data collection, ongoing supervision was done by the principal investigator on the selected sites during the data collection period. A checklist was used to assess the status of VCT service in relation to adequately trained staff and whether they fulfilled all the minimum packages for VCT service as indicated in the Ministry of Health guide line.

\section{Data processing and analysis}

The recorded interviews were transcribed from the tape recorder, and translated from the local language into English. The translated written document was coded; sorted and analyzed by the principal investigator. The quantitative data was entered, cleaned, and analyzed by using statistical package for the social sciences (SPSS) version 13.0 software. The qualitative part of the study was analyzed and interpreted by thematic areas. By using multiple regressions, the quantitative data was analyzed and interpreted, to know the relationship between the dependent and independent variables. The descriptive statistics were computed for most of the study variables and confidence interval and odds ratio were also interpreted.

\section{Ethical consideration}

Ethical clearance was obtained from a joint MPH program of UoG and Addis Continental institute of Public Health. Letters were written to the offices of Addis Ababa city administration Health Bureau, OSSA Addis Ababa branch, the Oromia Health Bureau, the Adama City Administration Health Department and OSSA Adama branch and other concerned bodies to obtain permission and cooperation for data collection. Study participant were briefed about the confidentiality of their responses, and the importance of providing the right information. Informed verbal consent was secured from the study subjects to participate in the study.

\section{RESULTS}

\section{The quantitative result}

By using a cross-sectional type of quantitative study, an attempt was made to assess the level of client satisfaction with mobile VCT services in Addis Ababa and Adama cities. The response rate was $100 \%$ from 279 client respondents, and among these 126 (45\%) and 153 (55\%) were from Addis Ababa and Adama OSSA branches respectively.

\section{Socio-demographic and socioeconomic characteristics of clients}

Out of the total number of espondents, 233 (83.5\%) were males and $46(16.5 \%)$ were females. The majority of the clients $(175 ; 62.7 \%)$ were in the age group of 20 to 29 years. The mean age of the client respondents were 24.5 years old. The educational level of respondents varied from illiterate to degree holders being dominated by $140(50.2 \%)$ junior and secondary school levels (grades 7 to 12$)$ and next to this $78(27.9 \%)$ elementary school level (grades 1 to 6 ). Concerning the type of jobs that the respondents had, there were different kinds of occupations. But, the majority was students while the rest were engaged in a variety of odd occupations (Table 1).

\section{Sexual characteristics of the respondents}

Of the 279 client respondents, $76 \%$ of them had the experience of sexual intercourse. The mean age of their first sexual intercourse was 19.5 years which ranged from 15 to 24 years. $71(36 \%)$ of the respondents had the experience of multi partner sexual behavior. $43(20.8 \%)$ of male respondents ever had sexual relation with commercial sex workers. Regarding condom use, 56 $(27.9 \%)$ of the respondents had never used condom and $97(43.31 \%)$ always used condom. With regard to the assessment of respondents' risk for acquiring human immunodeficiency virus infection and acquired immune deficiency syndrome (HIV/AIDS), 74 (26.5\%) clients believed that they were at risk while 149 (53.4\%) believed that they were not at risk.

\section{Source of information and utilization of mobile VCT service}

Most of the respondents 98 (34.4\%) and 69 (25.7\%) heard about the mobile VCT service from neighbors and friends, respectively. The majority of respondents 233 (82\%) were referred by themselves, and $40(14.3 \%)$ were recommended by others (friends, health personnel and sexual partner). The reasons for using the service 197 (72.2\%), 27 (9.9\%) and 21 (7.7\%) were "to know myself', "suspect myself" and "for marriage", respectively. 143 $(51.8 \%)$ of the clients were repeat testers and 120 $(43.5 \%)$ of them were new testers. The convenient times for most of the respondents $(76 ; 27.2 \%)$ to use the service were from Monday up to Friday (6 am to $8 \mathrm{pm}$ ) and $72(25.8 \%)$ of them were from Monday up to Friday late afternoon (5 to $8 \mathrm{pm}$ ).

\section{Reason for preferring the mobile service and duration of stay in the service site by clients}

Seventy two percent of the respondents met their counselors immediately after they arrived at the mobile service site, and $28 \%$ after 30 minutes. $69.5 \%$ of the respondents received their test result within 30 min time, and $27.2 \%$ received from 30 to $60 \mathrm{~min}$ and the 
Table 1. Comparison of mobile VCT service satisfaction of clients by VCT site, socio demographic characteristics and time variables in Addis Ababa and Adama, Ethiopia, February, 2010.

\begin{tabular}{|c|c|c|c|}
\hline \multirow{2}{*}{ Variable } & \multicolumn{2}{|c|}{ Satisfaction } & \multirow[b]{2}{*}{ COR $(95 \% \mathrm{Cl})$} \\
\hline & Yes & No & \\
\hline \multicolumn{4}{|l|}{ VCT site } \\
\hline Addis Ababa & 123 & 2 & 1.00 \\
\hline Adama & 151 & 3 & $1.222(0.201,7.429)$ \\
\hline \multicolumn{4}{|l|}{ Religion } \\
\hline Christians & 247 & 3 & 1.00 \\
\hline Others & 27 & 2 & $6.099(0.976,38.124)$ \\
\hline \multicolumn{4}{|l|}{ Ethnicity } \\
\hline Amhara & 110 & 1 & 1.00 \\
\hline Oromo & 122 & 3 & $2.705(0.277,26.387)$ \\
\hline Others & 42 & 1 & $2.619(0.160,42.834)$ \\
\hline \multicolumn{4}{|l|}{ Marital status } \\
\hline Ever married & 93 & 2 & 1.00 \\
\hline Never married & 181 & 3 & $0.771(0.127,4.693)$ \\
\hline \multicolumn{4}{|l|}{ Income } \\
\hline No income & 70 & 2 & 1.00 \\
\hline Has income & 204 & 3 & $0.515(0.084,3.144)$ \\
\hline \multicolumn{4}{|c|}{ Waiting time to receive result } \\
\hline$<30$ minutes & 202 & 4 & 1.00 \\
\hline$>31$ minutes & 72 & 1 & $0.701(0.077,6.379)$ \\
\hline \multicolumn{4}{|c|}{ Duration of counseling } \\
\hline$<15 \min$ & 97 & 1 & 1.00 \\
\hline $16-30 \mathrm{~min}$ & 122 & 2 & $1.59(0.142,17.797)$ \\
\hline$>31 \mathrm{~min}$ & 55 & 2 & $3.527(0.313,39.792)$ \\
\hline
\end{tabular}

remaining $3.2 \%$ after 60 min. Regarding the duration of counseling sessions (their stay with counselors), the majority of the clients $(112 ; 40.1 \%)$ being responded from less than 15 min and next to these $111(39.8 \%)$ were 15 to $30 \mathrm{~min}$ and the rest stayed greater than $31 \mathrm{~min}$.

\section{Willingness to share HIV test results and mobile VCT service satisfaction by clients}

13. $4 \%$ of the respondents wished another counselor in regards to age and gender, and $95.8 \%$ of respondents were willing to share their result with someone (spouse, sexual partner, family, friends and neighbors). Most of the respondents (above 90\%) believed that they benefited and were satisfied with the competence of counselors, the confidentiality and privacy of the counseling rooms, the duration of the counseling sessions and the respect they got from their counselors. The overall mobile VCT service satisfaction of the respondents was $98.2 \%$.

\section{RESULTS}

In addition to the quantitative study type of method, this study has used a qualitative type of study method. Hence, by using this method, the competence of the counselors and the service delivering process of the mobile VCT services were assessed. The competence of counselors was assessed by in-depth interviews with five counselors who had two and above years of counseling experience. There were two counseling observation 
sessions in each study area.

\section{Summary results from in-depth interviews with counselors and managers}

The interest of counselors shown towards counseling, all of them have an interest towards in their work. Most of them had a training of two weeks and some of them more than that time. Even though they believed that the training they had initially was relevant for the counseling work, there should be ongoing counseling trainings. They had a counselor support group and they believed also that the support group could help them in their challenges. In Adama OSSA branch, though the counselors counseled up to 30 clients per day, they didn't feel that they were overburdened whereas the counselors found in Addis Ababa OSSA branch said that they counseled a maximum of 20 clients per day and they felt that they were overburdened.

Regarding privacy and confidentiality of clients in Adama, they used tents as counseling rooms. These rooms had decent comfort and accommodated privacy and confidentiality of clients. But in Addis Ababa, they used partitions of a truck as counseling rooms. So the rooms were very narrow and the counselors cited that the counseling rooms can keep privacy and confidentiality, in terms of comfort, the counseling rooms were very narrow and became very hot during summer time. But one counselor didn't deny that there was the possibility of the conversations that was made by counselors and clients during counseling sessions were heard by others.

Consequently, these hot rooms made the counseling sessions less convenient for the counselors and the clients. One of the counselor respondents complained:

"Since the ventilators of these rooms did not function properly, the rooms became very hot during summer time. As a result of the high temperature of these rooms, some clients asked us: "As we couldn't resist the heat of the rooms even for this short period of time, how can you resist staying in this hot room the all day?"Actually I have also observed some clients who sweated during the counseling sessions. And those who couldn't attend the counseling sessions properly wanted to get out of the counseling rooms hurriedly."

As regards the duration of counseling sessions, since each client has different levels of sexual behavior, most of the counselors said that they did not give equal time for every client. This is because clients who did not have any risky sexual behavior and when tested for HIV repeatedly, did not need much time as indicated in the ministry of health $(\mathrm{MOH})$ guidelines. One of the counselors complained by saying:

"Even though I tried to counsel according to the standard time of counseling session, especially repeat testers did not accept me." Surprisingly, one client said to me at one time "since I have been tested repeatedly, I know well about what you are talking, so why do you waste my time?"

The counselors did not deny the fact that there is the possibility of becoming positive in clients who did not have any sexual risk behavior and clients who had negative results in their previous tests. As a result of this, they confirmed that although the clients did not have any risky sexual behavior during risk exploration in the pre test counseling session, they gave enough time to prepare the clients for the test. On the other hand, knowledge of the clients determine the duration of the counseling session. Counselors found in Adama OSSA branch said that there is a difference in knowledge about HIV/AIDS between rural and urban population. Consequently, they did not give equal time for these two different groups. Since the clients who are found in rural areas have less knowledge than the urban ones, they used to give more time to them during the counseling sessions.

Regarding to the counseling protocol, in Adama OSSA branch, there was a counseling protocol in each counselor's table, and they used it during counseling sessions whereas in Addis Ababa OSSA branch there was none in all counselors' tables or any other place in the counseling rooms.

With reference to the annual plan made by VCT managers in both study areas, their plan was based on the number of counselors that were found in their mobile VCT sites. And by using the MOH VCT guidelines, a counselor was supposed to see about 10 to 15 clients per day. If counselors have got clients more than 15, they have to inform their managers in order to arrange additional counselors, counseling rooms and all the necessary materials needed. But in practice, as revealed in the above paragraph, particularly a counselor found in Adama OSSA branch counseled up to 30 clients per day. So, this shows that there might be a communication gap between counselors and their managers or the supervisors might not make a regular follow up to the high number of clients counseled per day by the counselors.

In Adama OSSA branch the managerial officials said that before they went to a new service rendering site, they always communicated with Wereda Health Desk and the HIV focal person found in that wereda. They selected a convenient service rendering site in that particular area together with the focal person. This focal person also mobilized the community during different community gatherings. In addition to these, starting from the service rendering day they mobilized the community by using a car in different parts of that particular Wereda and they posted different types of messages about 
HIV/AID on the tents. But, in Addis Ababa OSSA branch, they didn't have such kinds of work integration with the Kebele health desks and HIV focal person found in kebele levels. They promoted the service by using only different illustrations about HIV/AIDs found on the truck and sometimes they also used a microphone found on the top of the truck to mobilize the community.

Concerning the referral system of the two service areas both had a problem of feedback from the health institutions that received the referred clients. In Adama OSSA branch, there were few areas that were far from antiretroviral treatment (ART) clinic and also had a high percentage of positive clients. In such cases, even though the counselors referred the clients, who had positive test results to those ART clinics, situated far away from the service delivering sites, the clients couldn't go there due to different socio-economic and transportation problems.

\section{Results from observation of counseling sessions and observation of the check list}

Four session observations were done in both areas. There were six counselors and among them two counselors were community counselor and the rest four had a nurse's back ground. The session observations had been undertaken by the principal investigator. Fortunately, the test result of all the clients who were counseled during counseling session observations were negative. Consequently, there was no chance to get clients who had positive test results.

As mentioned earlier, in Addis Ababa OSSA branch, the counselors did not have any counseling protocol on their tables or on any other place in the counseling rooms. As a result, the study has observed that they missed some steps which were found on the counseling protocol. In all the observations, at the beginning of the counseling sessions, all the counselors introduced themselves and explained about the whole process of the counseling including the confidentiality of the test results and any other information about the clients. But the exploration of the options for reducing risk, three of the counselors missed identifying the obstacles for risk reduction and two of them missed the assessment and enhancement of condom skills.

The time that the four counselors spent during the pre counseling sessions was ranged from 11 to $15 \mathrm{~min}$ and the post counseling was from 3 to 5 min whereas, the standard duration for the pre and post counseling sessions was 30 to $40 \mathrm{~min}$. In general, except the above mentioned missed counseling steps and the short duration of counseling sessions, all counselors were found polite and had a good respect for their clients. In addition to these, they had good counseling knowledge and were competent in all other components of counseling sessions.

From the observation of the check list, both VCT sites had three counselors, one laboratory technician, one receptionist, a cleaner and a guard in each service area. According to the managers, if too many clients were coming to their site, they always used to recruit additional counselors temporarily in order to alleviate the work burden of the permanent counselors. There were five rooms three for the counseling, one for the reception and one for the laboratory in each study area. The counseling rooms that were available, in Addis Ababa OSSA branch were partitions of a truck, and their total area were less than four square meters which was less than the set standard( above nine square meters).

Regarding the communication materials that they had in the service areas, there were different types of posters posted on the outer parts of the truck and the tents. In Addis Ababa OSSA, there was no brochures and leaflets that were given to the clients while they were waiting their turn to see the counselors during the data collection period. In fact, the counselors said that they were providing these communication materials before that time but during data collection time it was out of stock.

Both service sites used the type of rapid test called KHB and STAT PAK. It enables clients to know their test result within $30 \mathrm{~min}$ after they gave their blood sample. The laboratories that were found in both study areas had the minimum packages that enable them to render the test services. To ensure the quality of the test in Addis Ababa OSSA branch, they had no any external quality control since the recent test kit (KHB and STAT PAK) was started. But in Adama OSSA branch, the laboratory technicians went periodically to the regional laboratory to evaluate their test skills. The regional evaluators gave them blood samples to do the test in their laboratory.

\section{DISCUSSION}

This study tried to assess the quality of mobile VCT services in Addis Ababa and Adama OSSA branches. The two mobile VCT services were under OSSA Ethiopia. Both rendered the service by moving to different places in the cities and the surroundings of Weredas.

There was no significant association found in this study between the overall service satisfaction of clients and the socio demographic characteristics and other study variables. But there was significant associations found in other study between overall service satisfaction with age, marital status and occupation. This might be explained by the sample size of this study is much lower than the facility based study (422). On the other hand, there was a difference in service approach and consequently in this study the satisfaction rate was very high. However, there was a remarkable difference found in percentiles between some variables in this study and variables of 
other studies (Dawit 2006).

Most of the respondents were in the age group of 20 of 29 years old. The majority of the respondents in this study $(83.5 \%)$ were male clients whereas $16.5 \%$ were female clients. The number of the female clients was very small compared with the facility based study in Addis Ababa which was $60.5 \%$. This also coincides with the rapid assessment by PSP- E which was done on mobile VCT clients. The study showed that females responded by saying that they were busy with household activities and did not get privacy during the day time as the mobile VCT service was rendered in open spaces (Dawit 2006; Makoane et al 2008).

Most of the respondents (75\%) never had sexual experience in their life time. $36 \%$ of the respondents were multi partners. This was also higher than the facility based study which was $16.3 \%$. $20.8 \%$ of male respondents never had sexual intercourse with commercial sex workers. This result coincides with the strategy which is found on the VCT guidelines that the mobile VCT service can target high risk groups (Asingwire 2004; Dawit 2006).

Most of the client respondents $(31.9 \% ; 28 \%)$ preferred the mobile VCT services site because they believed that the service provider kept their discussion confidential, and the service site was near their homes. This result is strengthened by the in-depth interviews with clients in this study. The majority of client respondents said that they preferred the mobile VCT service site as the service providers were not the permanent residents of that particular service area, and because of this reason the service providers were strangers and did not know each other and they believed that their confidentialities were kept secret. The counselors also confirmed that there were clients who waited for the mobile VCT service to be tested by. But this result is different from the facility based study except that the second reason of the clients in this study resembled the first reason of the facility based study, and in that study most of the respondents (43.9\%; 20.4\%) preferred it because the service site was near their homes and that the service was affordable or free re (Dawit 2006).

$62 \%$ of the client respondents met their counselors immediately after they arrived at the service site, and $73.8 \%$ of them received their test results within $30 \mathrm{~min}$. These percentages were lower than the facility based study which was 81.9 and $80.2 \%$, respectively.

Concerning the duration of time, 35.1 and $44.4 \%$ of the clients responded that the duration of the time they stayed with the counselors during the pre and post counseling sessions was $<15 \mathrm{~min}$ and 16 to $30 \mathrm{~min}$, respectively. This is also different from the facility based study in which 19.1 and $43.4 \%$ clients responded $<15$ and 16 to $30 \mathrm{~min}$, respectively. Obviously, this is also lower than the standard (30 to $40 \mathrm{~min}$ ) which was set by the MOH VCT guidelines. This time difference may be explained by the difference between the two types of the service approach. Since the mobile VCT service was provided on the road sides and other places where many people were found (market places, city squares, etc.), there were a high number of clients who came to be tested. Thus, in order to fulfill the test demand for this high number of clients, the counselors might be obliged to counsel hurriedly (Dawit 2006).

One of the factors that can affect the quality of VCT service is convenient time for the clients to be tested. In this study 27.2 and $25.8 \%$ of the clients chose their convenient time to be from Monday to Friday (6am to $8 \mathrm{pm}$ ) and from Monday to Friday late afternoon (5 to 8 $\mathrm{pm})$, respectively. This is strengthened by the in depth interviews with counselors and VCT managers in that there were times when they extended the service hours until $2 \mathrm{pm}$ during the nig ht time and also in some areas by starting the service early in the morning (7am) including weekends.

Out of the whole, about $95.8 \%$ of the client respondents had the willingness to share their test result to someone. This is also higher than the study done four years back in Addis Ababa which was $84.4 \%$. This may be explained by the fact that at this time there is an increase in awareness about HIV/AIDs among clients, and consequently there is a decrease in stigma and discrimination in the community (Dawit 2006).

The overall service satisfaction of the clients was found to be $98.2 \%$ in this study. This is very high compared with another similar study (79.2\%) which was carried in Addis Ababa. This may be explained by the fact that in the first place, the mobile VCT service delivery approach is different from the facility based VCT service. As mentioned earlier, the services were provided on the road sides and other places where many people were found. Even though clients had the need to get tested, probably they couldn't get the service due to certain factors like lack of time, the unavailability of the service near their homes or the confidentiality issues in the facility based VCT sites. And given the chance, the clients can use these services coincidentally on their way to market, working place and other places as well. Such conditions could create good opportunities for VCT clients to be tested. On the other hand, client satisfaction can be determined by service outcomes like clients having negative test results. As a result of all these factors, client satisfaction became much higher in this study than other similar studies (Dawit 2006).

VCT clients have concerns about confidentiality and privacy. There is also evidence that assurance of confidentiality and trust facilitate disclosure of risk behaviors during counseling sessions. Counseling rooms should provide audio visual privacy. Conversation between clients and counselors should not be seen or overheard by others. On the contrary, the counseling rooms found in Addis Ababa OSSA branches were very 
narrow $\left(<4 \mathrm{~m}^{2}\right)$ and the area of the rooms were much narrower than the Addis Ababa Health Bureau standard which was $>9 \mathrm{~m}^{2}$. Because of these reasons, conversations between the counselors and their clients could be overheard by others. This situation could also hinder clients to disclose their risk behaviors to the counselors (Dawit 2006; AAHB, 2004).

According to the MOH VCT guidelines, a counselor has to counsel a maximum of 10 to 15clients per day. But in this study, a counselor counseled a maximum of 20 to 22 clients per day in Addis Ababa and up to 30 clients per day in Adama. This is much higher than the facility based study which was a maximum of 12 clients per day. This work burden could cause a burnout process in the counselors and that a higher number of them might be inclined to seek withdrawals. One of the counselors did not hide the fact that sometimes they felt overburdened and consequently over stressed.

In addition to this, the counselors couldn't get enough time for the counseling sessions in order to cover the main areas of counseling. If they couldn't cover the main areas of the counseling session, clients couldn't get quality counseling. Thus, clients can obviously face stigma and discrimination (Dawit 2006; Asingwire 2004; Suman 2009).

In Adama OSSA branch, in some areas which had a high number of positive clients, there were no ART clinics near the service site. Even though the counselors referred these clients to far off places where ART clinics were found, clients couldn't go there due to transportation and other socio-economic problems. Consequently, this situation might make positive clients hopeless and bring negative outcomes. If positive clients couldn't get follow up treatment and support, they might not bring positive behavioral changes. Without support, some HIV positive clients become so distressed or angry that they increase their sexual risk behavior and/or suffer depression and anxiety (Suman 2009).

Regarding the external quality control of HIV test, when the mobile sites used the previous test kit (Determine, Unigold and Capilus), they sent blood samples to the regional laboratories. During the data collection period, both mobile VCT sites used a new test kit called KHB and STAT PAK. To do an HIV test by the recent HIV test kit, only some drops of blood is enough from a finger tip. Because of this practice of not taking much blood as in the previous test kit, the VCT service sites stopped to send blood samples to the regional laboratories in both areas. But, in Adam OSSA branch, the laboratory technicians went to the regional lab, to do HIV test from the blood sample that were given to them by the regional evaluators.

This is a good experience to know the test skills of the lab technicians, but it lacks the specificity and sensitivity test which could enable us to know the validity of the testing algorism.

\section{CONCLUSION}

The overall service satisfaction by the clients was very high in this study. And there was no significant association between overall service satisfaction with the different study variables. In addition to government working hours, extra working time (early in the morning, late afternoon and lunch time) were chosen by most of the respondents. Short duration of counseling time; a high number of clients counseled by counselors per day, inconvenient counseling rooms and absence of counseling protocol in the rooms; insufficient promotional activities; a feedback problem from the health institutions and lack of external quality control of the tests were findings from the qualitative study which can affect the quality of the mobile VCT services.

Regarding the competence of the counselors, they were found to have good respect for their clients, and had good counseling knowledge except that sometimes they missed some counseling steps in the counseling protocol.

\section{RECOMMENDATIONS}

The following points were recommended:

1. OSSA Ethiopia should always make periodical supervision on the mobile VCT sites in relation with the duration of the counseling time, the number of clients seen by the counselors per day in the sites, the availability of counseling protocol in all the counseling rooms and check whether counselors follow the protocol or not during every counseling session.

2. The area of the counseling rooms found in Addis Ababa OSSA branch should be improved by partitioning the truck only into two counseling rooms and adding another tent room as a third counseling room.

3 . If the service rendering hours can be extended in the late afternoon (5 to $8 \mathrm{pm}$ ) and kept open during lunch time, the service can reach many people who have no time during government working hours and days.

4. By creating strong work integration with health institutions and other NGOs, OSSA Ethiopia can improve the referral linkages and the feedbacks from the health institutions which received the referred clients.

5. In order to mobilize the community and select a convenient place for the mobile service delivery, the Addis Ababa OSSA branch should make work integration with the Wereda and Kebele HIV focal persons.

6 . To ensure the quality of the test, the regional labs should make on - site evaluations in the mobile VCT service delivery sites at list once a year.

7. FHAPCO should take actions to improve the quality of the mobile VCT services.

\section{Conflicts of interest}

The authors have not declared any conflict of interest. 


\section{ACKNOWLEDGMENT}

The authors are grateful to the Addis Ababa and Oromia Regional Health Bureaus for giving them approval of this study, and communicating to the Addis Ababa and Adama OSSA branches. Their special thanks also goes to the mobile VCT site managers, VCT counselors, and clients who participated in the study.

\section{REFERENCES}

Addis Ababa health Bureau (AAHB) (2004). VCT service Guideline. Asingwire N (2004). Feasibility(Study of The Mobile Van for Voluntary counseling and testing for HIV/AIDS, Dept. of Social Work \& Social Admn. Makerere University, Kampala-Uganda.

CDC (Centers for Disease Control and Prevention), Division of HIV/AIDS Prevention, Basic statistics (2004). Available at: http://www.cdc.gov/hiv/pdf/statistics_2004_hiv_surveillance_report_v ol_16.pdf
Joseph D, Waikars DS, Buch von Schroeder C, Khan G (2006). Mobile Voluntary Counseling and Testingfor HIV to increase access among high risk groups in Rwanda and India, International AIDS Society 16 Abstract No. WePE0357

Dawit A (2006). Assessment of Quality of Voluntary HIV Counseling and Testing Services in Addis Ababa, A. A. University, Ethiopia.

Makoane C, Sanjana P, Leykun, A (2008). Operational Manual for Implementing HIV Mobile Counseling and Testing Services in Ethiopia. Bethesda, MD: Private Sector Partnerships-Ethiopia, Abt.

Suman M (2009). HIV/AIDS coordinator, Technical support division, UNFPA.

WHO (2002). Increasing access to knowledge of HIV status: conclusions of a WHO consultation.

WHO, UNICEF, UNAIDS (2009). Towards universal access: scaling up priority HIV/AIDS interventions in the health sector, progress report.

WHO (2009). Models of VCT service delivery. Available at: http:// www. Searo WHO.Int/Link Files // training materials voluntary-module5-2. 